\title{
ARTICL Opportunities for enhancing brain health across the lifespan
}

\author{
Alan J. Gow (10)
}

\begin{abstract}
Alan J. Gow, PhD, is Professor of Psychology and Deputy Director of the Centre for Applied Behavioural Sciences at Heriot-Watt University, Edinburgh, UK. His research explores the factors that predict healthy ageing, primarily focusing on the lifestyles and behaviours associated with brain health that might be potential targets for 'real-world' interventions (www.healthyageing. hw.ac.uk).

Correspondence Alan J. Gow. Email: A.J.Gow@hw.ac.uk
\end{abstract}

First received 29 Jul 2020

Final revision 26 Feb 2021

Accepted 1 Mar 2021

\section{Copyright and usage}

(C) The Author(s), 2021. Published by Cambridge University Press on behalf of the Royal College of Psychiatrists. This is an Open Access article, distributed under the terms of the Creative Commons Attribution licence (http://creativecommons.org/ licenses/by/4.0/), which permits unrestricted re-use, distribution, and reproduction in any medium, provided the original work is properly cited.

\section{SUMMARY}

As we age, there are characteristic changes in our thinking, reasoning and memory skills (referred to as cognitive ageing). However, variation between people in the timing and degree of change experienced suggests that a range of factors determine individual cognitive ageing trajectories. This narrative review considers some of the lifestyle factors that might promote (or harm) cognitive health. The focus on lifestyle factors is because these are potentially modifiable by individuals or may be the targets of behavioural or societal interventions. To support that, the review briefly considers people's beliefs and attitudes about cognitive ageing; the nature and timing of cognitive changes across the lifespan; and the genetic contributions to cognitive ability level and change. In introducing potentially modifiable determinants, a framing that draws evidence derived from epidemiological studies of dementia is provided, before an overview of lifestyle and behavioural predictors of cognitive health, including education and occupation, diet and activity.

\section{LEARNING OBJECTIVES}

After reading this article you will be able to:

- Understand the broad cognitive changes associated with age and why a focus on the lifespan may be beneficial;

- Appreciate how individual variation in cognitive change across the lifespan supports multiple factors (genetic and environmental) as determinants;

- Acknowledge that no single factor is likely to be critical and that an approach highlighting many small effects is required.

\section{KEYWORDS}

Education and training; brain health; cognitive ageing; Ideterminants.

In a UK-wide survey of adults aged 40 and older, more than $70 \%$ of respondents were somewhat or very concerned about their cognitive health declining (referred to as changes in 'thinking skills' throughout the public survey). Although they expressed high levels of concern, there was cause for optimism as over $90 \%$ believed that maintaining or improving cognitive health was possible (Gow
2018; Vaportzis 2018), suggesting an openness to positive brain health messaging. That said, over $40 \%$ of respondents reported either being unsure or that they did not know the factors associated with cognitive health (see Box 1 for more on the What Keeps You Sharp? survey). Given that populations are ageing and that cognitive health is a growing concern for many (Jessen 2020), it is important to support accurate and clear brain health messages for adults throughout mid-life and old age.

This narrative review will explore some of the lifestyle and behavioural factors associated with cognitive ability and change, including those that might slow, reduce or delay decline or act as risk factors that predict poorer outcomes. In doing so, the focus is on 'normal' cognitive ageing; that is, the general and characteristic age-related changes in thinking, memory and reasoning skills (Hertzog 2008). For those in the clinical sphere, it might be asked why that is necessary or beneficial. It is important to note that the current attention to cognitive ageing versus dementia is not to diminish the experiences of those living with, or caring for people living with, dementia, and the impact that has on health and social care provision more broadly. Rather, this narrative review is proposed as a companion to those considerations. The prevalence of dementia is estimated at about $7.1 \%$ of adults, or 1 in every 14, over the age of 65 in the UK (Alzheimer's Society 2014); even with no change in this (although there have been indications of prevalence stabilising or even reducing slightly in some high-income countries), the absolute numbers are predicted to rise as a result of demographic ageing trends. It is therefore important to highlight opportunities for proactive and preventive strategies that might reduce the risk of dementia or, more broadly, promote brain health across the lifespan. A brief overview of cognitive ageing versus dementia is outlined in Box 2.

\section{Overview of cognitive ageing}

\section{Cognitive abilities}

Throughout this narrative review, the term cognitive ageing will be used to refer to changes in thinking, memory and reasoning skills 'as a process of 


\section{BOX 1 What Keeps You Sharp?}

What Keeps You Sharp? was a nationwide survey of beliefs about, and understanding of, cognitive ageing in the UK. The survey was based on similar work in the USA and elsewhere, for example the brain health surveys conducted by AARP (Skufca 2015; Mehegan 2017a, 2017b).

In brief, the What Keeps You Sharp? survey was completed by 3300 people across the UK aged between 40 and 98 years in late 2016 to early 2017. The intention was to explore awareness of the cognitive changes we might experience with age and, importantly, the factors people identified as potentially beneficial. The findings were to be used to support public engagement activities related to cognitive ageing, provide useful information for cognitive ageing researchers in terms of public understanding of the area, and support the development of brain health messages.

A range of findings have been reported from the survey, including, for example, the qualitative responses to the question 'What would be one piece of advice you would give to someone to maintain or improve their thinking skills with age?'. Three main themes were suggested in the analysis, specifically: keeping fit and healthy in mind and body; staying engaged with the world around you; and attitudes to prevent decline (Niechcial 2019).

An accessible report from the survey for use by older people, their families, community groups, the third sector and practitioners is available (Gow 2018). Elements of the survey were also used to create a 'How well do you know your brain?' quiz on Age UK's Staying Sharp webpage (www.ageuk.org.uk/ information-advice/health-wellbeing/mind-body/stayingsharp/how-well-do-you-know-your-brain/), providing an accessible route into their more specific information and advice on brain health. Those interested in the survey, including access to the data, can contact the author.

\section{BOX 2 Cognitive ageing and dementia}

When changes in thinking skills with age are discussed, for many people the term dementia comes to mind. In conversations or media reporting, dementia is often used as a synonym for what to expect as we get older. However, dementia is not a normal part of ageing. Dementia is a broad collection of symptoms characterised by problems with memory and other thinking and reasoning skills, and those problems mean that performing day-to-day tasks becomes more difficult. Dementia is caused by damage to the structure of the brain and the impact that has on its functions. That damage is caused by a range of diseases and the most common cause of dementia is Alzheimer's disease.

But dementia and 'normal' cognitive ageing are different. We might all experience some changes in our cognitive abilities as we age, but those are not necessarily the result of a disease process. For example, problems with short-term memory are common in dementia and in a real-life scenario this might be
'Forgetting the names of close friends or family, or forgetting recent events - for example, visitors you had that day'. There can also be memory lapses with normal ageing, but these might be more likely to be 'Sometimes forgetting people's names or appointments, but remembering them later'.

Similarly, language problems are seen in dementia, such as 'Having frequent problems finding the right word or frequently referring to objects as "that thing"', while normal ageing might be more like 'Having a bit of trouble finding the right word sometimes'.

Some good resources outlining differences between cognitive ageing and dementia are available on the Alzheimer's Society's 'Normal ageing versus dementia' webpage (from which these examples are taken), at www.alzheimers.org.uk/ about-dementia/symptoms-and-diagnosis/how-dementia-progresses/normal-ageing-vs-dementia. gradual, ongoing, yet highly variable changes in cognitive functions that occur as people get older' (Blazer 2015, p. 2). As Blazer et al continue, 'Cognitive aging is a lifelong process. It is not a disease or a quantifiable level of function'. Discussion of cognitive abilities, and the agerelated changes in them, encompasses a range of skills, from verbal and numerical abilities, attention and processing speed to reasoning and problemsolving, and the various aspects of memory, including working, semantic and episodic memory. The specifics of those are beyond the scope of this narrative review but good overviews are available (Gutchess 2020).
In terms of the range of thinking skills, however, a consistent finding replicated across hundreds of studies is that performance on measures of these are positively correlated, referred to as the positive manifold (Tucker-Drob 2019a). That is, someone who generally performs well at one type of task will likely perform well at others; note though, that this is 'generally' and 'likely', as different abilities have different intercorrelations, which allows the structure of broad cognitive domains to be defined. Although the structure of cognitive abilities has been debated, there is broad consensus of there being shared variance across diverse abilities, perhaps accounting for 
about $40 \%$, as well as domain-specific variance (Carroll 1993).

\section{What goes when?}

Although being defined broadly for current purposes, it is important to note that the trajectory of cognitive ageing varies by ability (Hedden 2004; Hertzog 2008). The question of when cognitive decline might become apparent is of central importance but is not necessarily simple to answer. Processing speed, for example, is suggested to peak in the early 20 s and decline gradually across the remainder of the lifespan. That relatively early peak and decline is atypical, as many skills continue to develop through mid-life (Hedden 2004; Blazer 2015). A useful distinction is often made between fluid and crystallised abilities. Fluid abilities are often defined as being more age sensitive and therefore decline earlier, perhaps indicating a biologically limited aspect of brain function (Hedden 2004; Tucker-Drob 2019a). Crystallised abilities, so named to engender a sense of their fixedness, are more resistant to age-related changes and continue to develop through mid-life and into old age, with vocabulary or semantic knowledge often cited as exemplars of such age-resistant abilities (Hedden 2004; Blazer 2015).

In addition, it is often discussed how much of the age-related change in cognitive abilities is due to shared variance across domains and how much is specific. A recent meta-analysis suggested that about $60 \%$ of the variance in cognitive change was shared, directing attention towards the mechanisms that might underly this 'general factor of cognitive aging' (Tucker-Drob 2019b), while simultaneously highlighting that some changes are specific to cognitive domains.

It is important to clarify that, when discussing cognitive changes with age, these are 'average' changes observed in studies of many hundreds, or indeed thousands, of participants. While we might all experience these changes, their extent and timing are variable. Indeed, it is the existence of this person-to-person variation that supports attempts to identify the potential determinants of cognitive ability level and change. Figure 1 illustrates that variation with four hypothetical people and 'cognition' as a broad term. The shading suggests a general area of opportunity; that is, across the lifespan we might have the potential to be performing better or worse at any given age depending on the influence of a range of factors. In the illustration, all four people start at the same level, but their patterns of cognitive development vary from early adulthood through mid-life and into old age. Person A, for example, appears to show continued

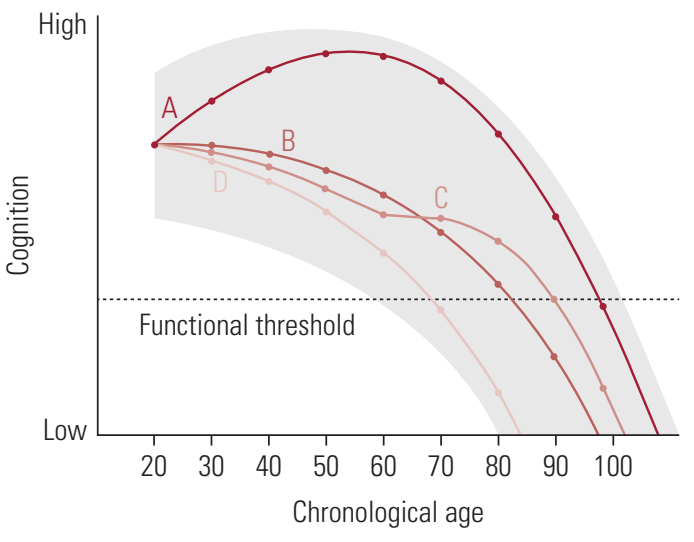

Depiction of the zone of possible cognitive development for a given individual, along with four developmental curves $(A, B, C, \& D)$ indicating specific possible outcomes. Each possible curve starts from the same functional level at age 20 , with different trajectories resulting as a function of interactions among behavioral, environmental, and genetic factors that permit vertical movement within the zone at different points in the life span" (Hertzog 2008). Reproduced with permission from SAGE Publications.

growth through mid-life, perhaps as the result of the opportunities presented by pursuing higher education followed by a mentally challenging occupation. Person C, meanwhile, appears to be declining slightly more than average from their 20s onwards, before a stabilisation in their 60s, perhaps as a result of retirement from a less challenging occupation followed bytaking up some new post-retirement opportunities (Hertzog 2008). A consideration of some of the lifestyle and behavioural factors that might determine better or poorer trajectories forms the basis of the second half of this review.

\section{Genes versus lifestyle}

Before exploring the determinants of cognitive ageing, and specifically potentially malleable determinants, it is useful to summarise the extent to which genetic factors might account for individual variation in cognitive ability level and change. Studies have estimated the heritability of cognitive ability to increase from around $20 \%$ in early childhood to $60 \%$ in adulthood (perhaps being as high as $80 \%$ in old age) (Plomin 2015). Although there is clearly a genetic component, research over the past decade using genome-wide association techniques has suggested that cognitive ability, as with many other traits, is 'highly polygenic' (Davies 2011). Or rather, that many thousands of genetic variants each make very small contributions: the 'largest effect sizes of associations are very small indeed [...] If the largest effect sizes are so small, 
the smallest effect sizes must be infinitesimal' (Plomin 2015). Even consortia combining results from over 50000 participants have accounted for just under $30 \%$ of the variance in general cognitive ability, suggesting ever larger samples will be required to identify ever smaller contributions (Davies 2015).

In discussing genetic contributions, it is important to distinguish between predicting individual variability in cognitive ability level versus change over time. In one study with measures of cognitive ability across the lifespan, for example, it was suggested that genetic factors might account for about $25 \%$ of the variability in cognitive change from childhood (age 11) to later life (age 70) (Deary 2012); the counterpoint, therefore, was that environmental factors might account for the larger proportion of the variability in cognitive change across the lifespan. In terms of changes during old age, research with the same sample did not find associations between polygenic scores and cognitive change from age 70 to 79 (Ritchie 2020). More recently in another longitudinal sample, researchers suggested that a polygenic risk score for late-onset Alzheimer's disease was associated with 25-year change in cognitive ability, although partly confounding mid-life and later-life changes, given the nature of the sample (Kauppi 2020).

Understanding the extent of the genetic contribution provides a useful focus: how much of cognitive ability level or change might we be able to influence, or rather, what is our target? In summarising briefly, then:

- Our cognitive abilities develop and change across the lifespan, including age-related changes in old age;

- The timing and extent of these changes vary by the type of ability;

- People also vary in the extent of age-related change they experience (and indeed the peak they might reach earlier in the lifespan);

- Person-to-person variation directs attention towards identifying the genetic and environmental determinants, with some evidence suggesting a stronger environmental contribution to lifetime cognitive change;

- And that directs attention towards "what keeps us sharp?'.

\section{No magic bullet}

Before discussing specific lifestyle factors, it is useful to consider a framing proposed by Corley et al (2018). In summarising findings from a long-term study of ageing where a number of factors have been identified as potential predictors of cognitive ability level and/or change, the authors proposed considering these in terms of 'marginal gains'. The marginal gains approach highlights that each individual predictor might account for a small (or indeed very small) proportion of variance and that these contributions may not be independent.

As Corley et al (2018) express it, the concept of marginal gains, often used in business and sports performance, therefore proposes that if you improve in every variable (or lifestyle factor) underpinning or influencing your performance (in our case, cognitive abilities) by just $1 \%$ or so, then, cumulatively, you get a significant improvement, or an "aggregate of marginal gains"'. To refer back to Fig. 1, rather than education, occupation or any other single factor accounting for the divergence in the four hypothetical trajectories, many dozens of factors and their interactions are likely involved.

\section{Reverse causation}

Although it is unlikely that any single lifestyle or behavioural factor is critical, there is one factor that accounts for a large proportion of the variance in cognitive ability in later life: earlier cognitive ability. There is a high degree of stability in cognitive ability across the lifespan. Not only that, but earlier cognitive ability is itself a predictor of many behaviours of interest throughout life. This leads to the issue of reverse causation. As an example, people reporting more participation in mentally engaging activity generally have higher cognitive ability, the suggestion often being that it is their engagement that has supported the development of their better cognitive abilities, helped maintain those or protected against age-associated decline. However, people who were more cognitively able to begin with are also those who become more active adults. Once that has been taken into account, the association between activity and cognitive ability in later life might be reduced or removed entirely (Gow 2012).

This issue of reverse causation obscures the search for predictors of cognitive ageing, but very few studies are able to account for this, partly as a result of not having cognitive data from across the lifespan (Bielak 2010; Gow 2012). In considering lifestyle factors that might be beneficial, we must be mindful that the associations reported are possibly overestimates. That provides further support for the suggestion that approaches to promoting brain health should be framed in terms of marginal gains (Corley 2018): small changes across a number of factors are likely to minimise the risk of, for example, making changes to just one factor where the results supporting that were particularly confounded by reverse causation. 


\section{Modifiable risk factors for dementia}

In focusing on cognitive ageing, it is important to acknowledge that many of the risk or protective factors may be the same as those for dementia. The underlying pathways resulting in dementia are varied, and a Lancet review (Livingston 2017) explored the population attributable fraction (PAF) associated with a range of potentially modifiable risk factors ('the percentage reduction in new cases over a given time if a particular risk factor were completely eliminated'). The report suggested that nine factors individually account for the PAF, ranging from $0.8 \%$ (obesity) to $9.1 \%$ (hearing loss), or rather 'around $35 \%$ of dementia is attributable to a combination of the following nine risk factors: education to a maximum of age 11-12 years, midlife hypertension, midlife obesity, hearing loss, late-life depression, diabetes, physical inactivity, smoking, and social isolation'. To provide some comparison for those figures for the modifiable factors, the authors suggested that if the e4 allele of apolipoprotein E (APOE) was no longer present, that could represent a 7\% decrease in dementia incidence (Livingston 2017). The review was recently updated, adding three further modifiable risk factors: excessive alcohol consumption, traumatic brain injury and air pollution (Livingston 2020). In total, it was estimated that the updated 12 risk factors might account for $40 \%$ of dementia cases globally.

A meta-analysis focusing on Alzheimer's disease considered the evidence for 104 modifiable lifestyle factors and 11 interventions, classified according to the quality of the evidence and strength of recommendation (Yu 2020). Similar to previous reviews (Winblad 2016; Livingston 2017, 2020), the authors proposed a multifactorial approach to risk reduction. They identified 21 evidence-based suggestions; 19 of these were classified as 'strong suggestions', 9 of which were rated with Level A evidence (evidence was rated as Level A, (strong), B (weaker) or C (weak); Yu 2020). These are illustrated in Fig. 2, showing the relative point in the lifespan that those factors may be most relevant.

Considering both the full lifespan and a broad range of factors is therefore critical (Barnes 2011; Winblad 2016; Livingston 2017; Yu 2020). For example, Barnes and Yaffe (2011) estimated that, if the prevalence of seven risk factors (diabetes, mid-life hypertension, mid-life obesity, smoking, depression, cognitive inactivity or low educational attainment, and physical inactivity) were $10 \%$ lower, there would be as many as 1.1 million fewer people with Alzheimer's disease worldwide.

Being able to quantify the potential to reduce the incidence of dementia from these reviews of numerous, large epidemiological studies offers optimism for the future. It is important to be clear, however, that this relates to population-level rather than individual risk.

\section{What do people think promotes cognitive health?}

Before closing this narrative review with a summary of some of the lifestyle and behavioural factors associated with cognitive ageing, it is useful to consider what people believe might be beneficial. In the UKwide survey referred to earlier (Box 1), respondents were provided with a list of activities that might commonly be expected to protect or promote brain health (although ranging in the robustness of the evidence that exists). Of those, the top five factors selected as beneficial were having a purpose, eating a healthy diet, mental challenge, good sleep and physical activity (Vaportzis 2018). Interestingly, however, while respondents reported engaging in a number of these activities regularly, this was rarely for the purposes of promoting brain health.

\section{Brain-healthy lifestyles}

The final section of this review considers a limited selection of the lifestyle and behavioural factors that might be relevant to cognitive ability and change across the lifespan. The factors considered are not exhaustive, and indeed, as discussed earlier with reference to 'marginal gains' (Corley 2018), no factor should be considered in isolation or as being given precedence. For each factor considered, a recent review(s) is briefly summarised, with individual studies providing additional context where appropriate.

\section{Education}

Education is associated with higher cognitive performance, but the effect of education as a determinant of cognitive ageing trajectories has been less clear (Seblova 2020). A recent review suggested that education might increase cognitive ability, reported as an increase of 1-5 IQ points per additional year of education (Ritchie 2018). Interestingly, the benefit of education was suggested as persisting across the lifespan, supported by a review by Chapko et al (2018) in which education was one of the few consistently reported determinants of cognitive reserve.

There is less certainty about whether education modifies the rate of cognitive change in old age. The most recent meta-analysis described education as having a negligible effect on cognitive ageing, such that 'the association of education with change is at least 12 times smaller than the corresponding association with level' (Seblova 


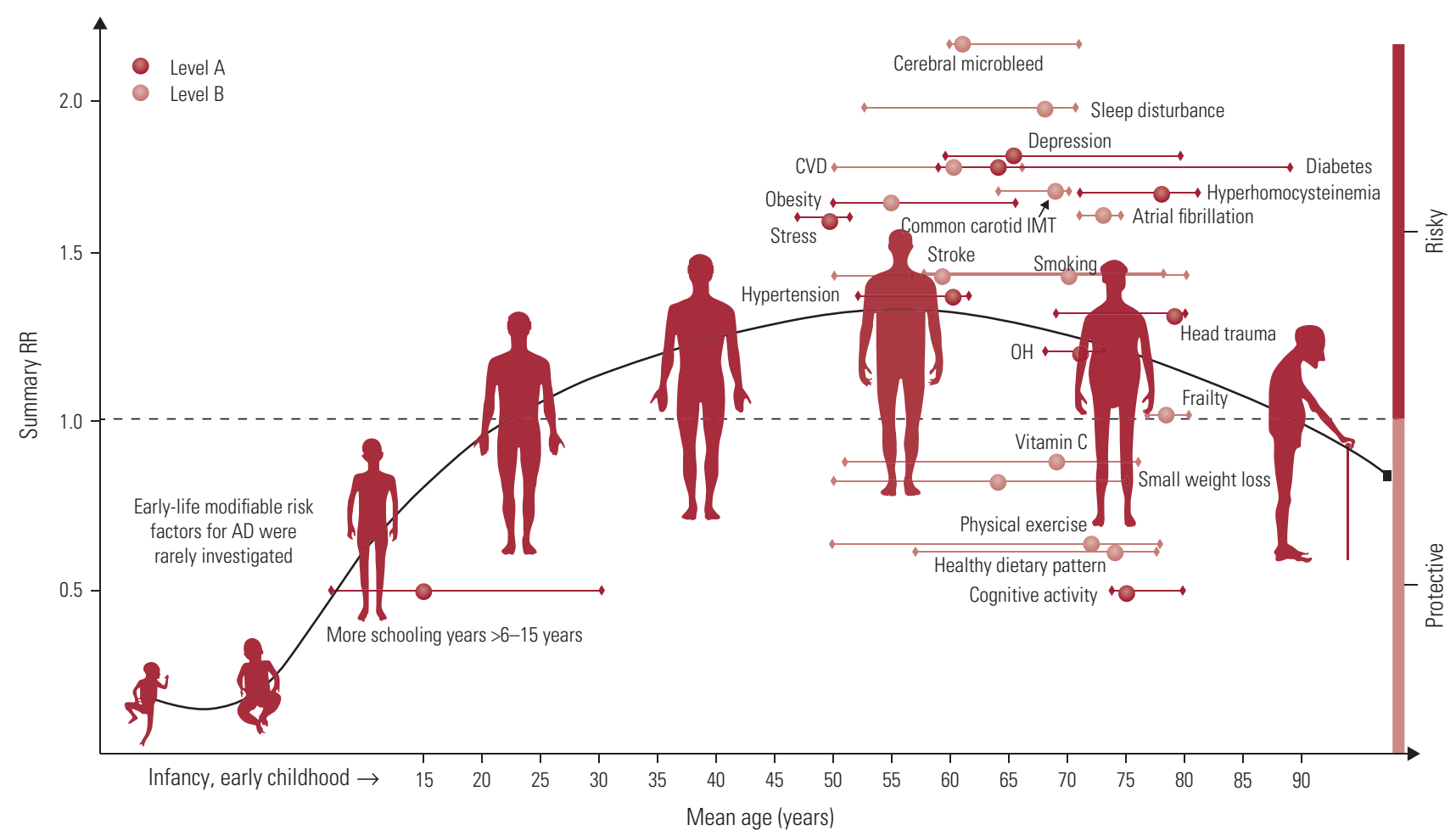

Distribution of modifiable factors with Class I recommendation throughout the course of life. Class I suggestions (benefit >>risk due to intervention) risk factors include 10 factors with Level A evidence (cognitive activity, hyperhomocysteinaemia, increased BMI in late life, depression, stress, diabetes, head trauma, hypertension in midlife, orthostatic hypotension and education) and 9 factors (obesity in midlife, weight loss in late life, physical exercise, smoking, sleep, CVD, frailty, atrial fibrillation and vitamin C) with Level $B$ evidence. The $x$ axis represents the mean age of the total sample (solid circle) with a range of mean age (short horizontal line) for observational prospective studies included. The y axis represents the summary relative risk (RR). AD, Alzheimer's disease; $\mathrm{OH}$, orthostatic hypotension; CVD, cerebrovascular disease; IMT, intima-media thickness. Reproduced from the Journal of Neurology, Neurosurgery \& Psychiatry, Yu et al (2020), with permission from BMJ Publishing Group Ltd.

2020). That is not to say that education is not relevant to cognitive health in old age: as it would be considered one of the factors that support development across the lifespan, education's role may be in providing a higher starting point from which later change might occur (Chapko 2018; Ritchie 2018; Seblova 2020).

\section{Occupational complexity}

The complexity of one's occupation is often considered as a potential determinant of later cognitive ability level and change. It is, however, important to reiterate that occupational complexity is likely to be an outcome of earlier ability, education and a range of other social determinants. That said, the complexity of work with people and data might be associated with cognitive ability level after controlling for those factors (Smart 2014). However, studies that have followed participants through the retirement transition have noted that any benefit of occupational complexity may be lost post-retirement (Finkel 2009). That is, continued engagement might be important in terms of maintaining any cognitive benefits observed.
Chapko et al (2018) suggested that the impact of occupation might best be described as inconsistent, perhaps as a result of the differential effects before and after retirement. For example, some studies have provided support for occupational complexity being protective against decline (Andel 2015), whereas others have not (Finkel 2009). That is not necessarily suggestive that higher complexity is a predictor of decline per se, but rather that those who have achieved a higher level of ability as a result of their occupational engagement may appear to experience faster change once that stimulation is withdrawn (Hyun 2019).

\section{Smoking}

Among the lifestyle factors consistently cited as being harmful to cognitive abilities is smoking. That said, a meta-analysis reported that the effect on cognitive decline was not significant (Peters 2008), whereas an earlier review reported greater decline in smokers (Anstey 2007). Although smoking status may not be associated with the rate of decline, more recently reported in Deal et al (2020), that is not to say that smoking is not 
The Global Council on Brain Health (GCBH; www.aarp.org/ health/brain-health/global-council-on-brain-health/) is an international collaboration, founded by AARP in the USA in partnership with Age UK. The GCBH brings together groups of 'issue experts' on factors relevant to brain health to distil the evidence and produce accessible reports. Each report provides an overview of the literature (although these are not intended as systematic reviews), including the knowns and unknowns, and where possible makes recommendations for older people, community organisations, policymakers, clinicians and practitioners. Each report is also accompanied by a 1-page infographic to support dissemination of the key messages.
Over 5 years, the GCBH has produced 10 reports covering a range of potential determinants of brain health, including physical activity, social connections, sleep, nutrition and heart health. All GCBH reports and infographics are available at www.aarp.org/health/brain-health/global-council-on-brainhealth/resource-library/ and they provide useful material for communication with the public. For example, Age UK include a link to the GCBH reports from their Staying Sharp webpage (www.ageuk.org.uk/information-advice/health-wellbeing/ mind-body/staying-sharp//) and base many of their recommendations on that work. harmful to cognitive health. If, for example, there is a consistent association between smoking and a lower level of cognitive ability in later life, those who smoke will be declining from that lower starting point.

\section{Diet}

In terms of diet, it is common to see studies reporting specific nutrients as being beneficial for brain health. However, there are often inconsistencies in the findings reported, specifically between observational versus randomised controlled trials, and it would be difficult to produce a detailed list of recommendations from hundreds of individually considered nutrients. It is therefore useful, of course, that individual nutrients exist within the context of more easily understandable dietary patterns, and it is increasingly common to consider dietary patterns versus the 'reductionist approach' (Jennings 2020). Similar to the 'marginal gains' argument for lifestyle factors more generally, individual nutrients may make only small, and not necessarily independent, contributions; any potential benefit likely derives from their cumulative influence within particular dietary patterns (and then again, diet is one potentially protective factor among many).

Whole-diet approaches have often considered the Mediterranean diet or combinations of that with other diets specifically designed to reduce hypertension, for example. A review suggested that those most closely adhering to a Mediterranean diet were less likely to develop an incident cognitive disorder (Wu 2017), with one study estimating that each extra point adherence to the Mediterranean diet was associated with about 1.7 years fewer years of cognitive ageing (Shannon 2019). Jennings et al (2020) also highlighted that dietary patterns provide better targets for intervention than specific components.

Dietary supplements have also come under scrutiny as potentially beneficial for brain health.
However, a report from the Global Council on Brain Health (Box 3) suggested, among other statements, that it was not possible to 'endorse any ingredient, product or supplement formulation specifically for brain health, unless your health care provider has identified that you have a specific nutrient deficiency'.

\section{Mental, social and physical leisure activities}

In considering lifestyle and behavioural factors associated with cognitive health, engagement in mental, social and physical leisure activities is commonly proposed as beneficial (Hertzog 2008; Bielak 2010). Mental engagement is sometimes considered a key factor, even being framed in the adage "use it or lose it'. Briefly, that encapsulates the sense that the deployment of cognitive abilities in completing mentally stimulating activities (or indeed educational or occupational pursuits) is what builds cognitive capacity or reduces decline. That said, the specificallymentally challenging aspects may be more prone to confounding by reverse causation (Gow 2012). In general, however, it is often reported that more active individuals have a higher level of cognitive ability and a reduced rate of change (Hertzog 2008), although the latter aspect is less consistently supported.

Intervention studies have been employed to address some of those inconsistencies, but also to explore the potential causal pathways between a change in some aspect of activity and changes in cognitive ability. In reviewing 'real-world' interventions, it was suggested that benefits were clearest from physical activity, and more so for the cognitive abilities sensitive to age-related decline (Vaportzis 2019). In a more recent review combining leisure activity and other psychosocial aspects, it was suggested that 'an evidence-based intervention strategy to improve global cognition, memory and executive functioning would be a group therapy carried out for 
4 months or more, promoting regular (at least weekly) activity, involving aerobic or resistance exercise, with a cognitively demanding and/or creative component' (Whitty 2020).

There are, however, specific difficulties in providing summaries, given the diverse nature of activities and interventions considered. Direct comparisons, and therefore meta-analyses, are often challenging.

Studies of lifestyle or activity-based interventions are few, owing to the complexity of running them, but there is a large literature on cognitive training paradigms (often commonly referred to as brain training). Targeted cognitive training has been reported as beneficial; that is, people show improvement on the specific tasks trained. However, there remains debate, and controversy, in the field regarding what that means, both theoretically and indeed practically (Simons 2016). In conducting their review, Simons et al (2016) noted that methodological limitations restrict the utility of many studies. Furthermore, the Global Council on Brain Health (Box 3) offer the following careful statement:

\begin{abstract}
"Most commercial products marketed as "brain games" are not what the GCBH means when discussing the benefits of cognitive training. If people play a "brain game," they may get better at that game, but improvements in game performance have not yet been shown to convincingly result in improvements in people's daily cognitive abilities. There is insufficient evidence that improvements in game performance will improve people's overall functioning in everyday life. For example, we do not have evidence establishing that getting better at playing Sudoku will help you manage your finances any better' (Global Council on Brain Health 2017, p. 4).
\end{abstract}

Brain games may be fun for many, but, as suggested by the Global Council on Brain Health report, the weak or non-existent evidence for their benefits limits broader recommendations for them.

\section{Conclusions}

That there is variation in cognitive ageing suggests a range of factors determine this. In exploring lifestyles and behaviours associated with cognitive health, it is important to reiterate that the factors considered in this narrative review are not intended to be exhaustive or complete (further factors might have included sleep, social connections versus isolation or loneliness, alcohol, etc.). Many of the points discussed, however, are relevant to those other aspects of lifestyle - how confounded might the evidence be by reverse causation? - and even when consistent associations are reported, these are likely to be small and part of a network of factors that protect or harm the ageing brain.

As with many such summaries, therefore, it is not (and should not) be possible to end with a short list of easy tips. The detailed overview provided by $\mathrm{Yu}$ et al (2020) does, however, provide the most recent framework across a range of factors (summarised in Fig. 2), and although generated in the context of Alzheimer's disease it is applicable to cognitive health more broadly. Indeed, the life-course framing of Fig. 2 and the Lancet reviews (Livingston 2017, 2020) highlight an important point. Although dementia might be diagnosed in later life, the disease processes may have been active for 20 or 30 years. Given the stage the disease will have reached to enable a diagnosis, efforts are best directed towards prevention. And not only prevention per se, but the building of capacity in early adulthood and throughout mid-life. According to Livingston et al (2020), being able to eliminate the 12 risk factors identified from across the life course could result in a reduction of global dementia cases by $40 \%$. Although it is never too early to start promoting brain health, it is also likely that positive and proactive changes at any age can be worthwhile. Or rather 'It is never too early and never too late in the life course for dementia prevention' (Livingston 2020).

As has been highlighted throughout, the combining of multiple recommendations is probably the best approach to delay the onset of Alzheimer's disease (Yu 2020) or to promote cognitive and brain health (Corley 2018). Although that may appear more difficult in one respect - targeting many factors instead of one or a few - it perhaps offers a greater chance of success. People appear to be open to the opportunities for maintaining and improving thinking skills with age. The advice may need to be very clearly about making multiple small changes (but that are all manageable) rather than trying to make singular and perhaps less feasible changes. Although we cannot yet conclude that they will be beneficial on an individual basis, making these changes is not likely to cause harm (as long as the usual considerations for safety and appropriateness are taken into account in becoming a bit more physically active or changing some aspect of diet, for example). It may be that cognitive health across the lifespan is about slow and steady.

\section{Acknowledgement}

A.J.G. acknowledges the support of The Ageing Lab research team at Heriot-Watt University, Edinburgh, who contributed to the What Keeps You Sharp? survey.

\section{Author contribution}

A.J.G. conceived, drafted and revised the manuscript. 
MCO answers

1 b $2 d \quad 3 c \quad 4$ e 5 e

\section{Funding}

This work was supported by Velux Stiftung (grant numbers 1034 and 1034a).

\section{Declaration of interest}

A.J.G. was principal investigator on the What Keeps You Sharp? survey; he was also a contributor to one of the Global Council on Brain Health reports and has contributed to Age UK's Staying Sharp webpages, detailed throughout this narrative review. None of these has provided any financial incentive or conflict of interest.

\section{References}

Alzheimer's Society (2014) Dementia UK: Update 2nd ed. Alzheimer's Society (https://www.alzheimers.org.uk/sites/default/files/migrate/ downloads/dementia_uk_update.pdf).

Andel R, Silverstein M, Kåreholt I (2015) The role of midlife occupational complexity and leisure activity in late-life cognition. Journals of Gerontology, Series B: Psychological Sciences and Social Sciences, 70 314-21

Anstey KJ, Von Sanden C, Salim A, et al (2007) Smoking as a risk factor for dementia and cognitive decline: a meta-analysis of prospective studies. American Journal of Epidemiology, 166: 367-78.

Barnes DE, Yaffe K (2011) The projected effect of risk factor reduction on Alzheimer's disease prevalence. Lancet Neurology, 10: 819-28.

Bielak AAM (2010) How can we not 'lose it' if we still don't understand how to 'use it'? Unanswered questions about the influence of activity participation on cognitive performance in older age - a mini-review. Gerontology, 56: 507-19.

Blazer DG, Yaffe K, Liverman CT (eds) (2015) Cognitive Aging: Progress in Understanding and Opportunities for Action. National Academies Press.

Carroll JB (1993) Human Cognitive Abilities: A Survey of Factor-Analytic Studies. Cambridge University Press.

Chapko D, McCormack R, Black C, et al (2018) Life-course determinants of cognitive reserve (CR) in cognitive aging and dementia - a systematic literature review. Aging \& Mental Health, 22: 921-32.

Corley J, Cox SR, Deary IJ (2018) Healthy cognitive ageing in the Lothian Birth Cohort studies: marginal gains not magic bullet. Psychological Medicine, 48: 187-207.

Davies G, Tenesa A, Payton A, et al (2011) Genome-wide association studies establish that human intelligence is highly heritable and polygenic Molecular Psychiatry, 16: 996-1005.

Davies G, Armstrong N, Bis JC, et al (2015) Genetic contributions to variation in general cognitive function: a meta-analysis of genome-wide association studies in the CHARGE consortium ( $N=53949)$. Molecular Psychiatry, 20: 183-92.

Deal JA, Power MC, Palta P, et al (2020) Relationship of cigarette smoking and time of quitting with incident dementia and cognitive decline. Journal of the American Geriatrics Society, 68: 337-45.

Deary IJ, Yang J, Davies D, et al (2012) Genetic contributions to stability and change in intelligence from childhood to old age. Nature, 482: 212-5.

Finkel D, Andel R, Gatz M, et al (2009) The role of occupational complexity in trajectories of cognitive aging before and after retirement. Psychology and Aging, 24: 563-73.

Global Council on Brain Health (2017) Engage Your Brain: GCBH Recommendations on Cognitively Stimulating Activities. GCBH.

Gow AJ, Corley J, Starr JM, et al (2012) Reverse causation in activitycognitive ability associations: the Lothian Birth Cohort 1936. Psychology and Aging, 27: 250-5.

Gow AJ (2018) What Keeps You Sharp? A National Survey about What People Think about Their Thinking Skills! Heriot-Watt University (http:// www1.hw.ac.uk/mediaservices/pageflip/What_keeps_you_sharp/).
Gutchess A, Thomas AK (2020) The Cambridge Handbook of Cognitive Aging: A Life Course Perspective. Cambridge University Press.

Hedden T, Gabrieli JDE (2004) Insights into the ageing mind: a view from cognitive neuroscience. Nature Reviews Neuroscience, 5: 87-96.

Hertzog C, Kramer AF, Wilson RS, et al (2008) Enrichment effects on adult cognitive development: can the functional capacity of older adults be preserved and enhanced? Psychological Science in the Public Interest, 9: 1-65.

Hyun J, Katz MJ, Lipton RB, et al (2019) Mentally challenging occupations are associated with more rapid cognitive decline at later stages of cognitive aging. Journals of Gerontology: Series B: Psychological Sciences and Social Sciences [Epub ahead of print] 27 Sep. Available from: https://doi. org/10.1093/geronb/gbz122.

Jennings A, Cunnane SC, Minihane AM (2020) Can nutrition support healthy cognitive ageing and reduce dementia risk? BMJ, 369: m2269.

Jessen F, Amariglio RE, Buckley RF, et al (2020) The characterisation of subjective cognitive decline. Lancet Neurology, 19: 271-8.

Kauppi K, Rönnlund M, Nordin Adolfsson A, et al (2020) Effects of polygenic risk for Alzheimer's disease on rate of cognitive decline in normal aging. Translational Psychiatry, 10: 250

Livingston G, Sommerlad A, Orgeta V, et al (2017) Dementia prevention, intervention, and care. Lancet, 390: 2673-734.

Livingston G, Huntley J, Sommerlad A, et al (2020) Dementia prevention, intervention, and care: 2020 report of the Lancet Commission. Lancet, 396: $413-46$

Mehegan L, Rainville C, Skufca L (2017a) 2016 AARP Social Engagement and Brain Health Survey. AARP Research.

Mehegan L, Rainville C, Skufca L (2017b) 2017 AARP Cognitive Activity and Brain Health Survey. AARP Research.

Niechcial MA, Vaportzis E, Gow AJ (2019) People's views on preserving thinking skills in old age. Educational Gerontology, 45: 341-52.

Peters R, Poulter R, Warner J, et al (2008) Smoking, dementia and cognitive decline in the elderly, a systematic review. BMC Geriatrics, 8: 36

Plomin R, Deary IJ (2015) Genetics and intelligence differences: five special findings. Molecular Psychiatry, 20: 98-108.

Ritchie SJ, Tucker-Drob EM (2018) How much does education improve intelligence? A meta-analysis. Psychological Science, 29: 1358-69.

Ritchie SJ, Hill WD, Marioni RE, et al (2020) Polygenic predictors of agerelated decline in cognitive ability. Molecular Psychiatry, 25: 2584-98.

Seblova D, Berggren R, Lövdén M (2020) Education and age-related decline in cognitive performance: systematic review and meta-analysis of longitudinal cohort studies. Ageing Research Reviews, 58: 101005

Shannon OM, Stephan BCM, Granic A, et al (2019) Mediterranean diet adherence and cognitive function in older UK adults: the European Prospective Investigation into Cancer and Nutrition-Norfolk (EPICNorfolk) Study. American Journal of Clinical Nutrition, 110: 938-48.

Simons DJ, Boot WR, Charness N, et al (2016) Do "brain-training" programs work? Psychological Science in the Public Interest, 17: 103-86.

Skufca L (2015) 2015 Survey on Brain Health. AARP Research.

Smart EL, Gow AJ, Deary IJ (2014) Occupational complexity and lifetime cognitive abilities. Neurology, 83: 2285-91.

Tucker-Drob EM (2019a) Cognitive aging and dementia: a life-span perspective. Annual Review of Developmental Psychology, 1: 177-96.

Tucker-Drob EM, Brandmaier AM, Lindenberger U (2019b) Coupled cognitive changes in adulthood: a meta-analysis. Psychological Bulletin, 145: 273-301.

Vaportzis E, Gow AJ (2018) People's beliefs and expectations about how cognitive skills change with age: evidence from a U.K.-wide aging survey. American Journal of Geriatric Psychiatry, 26: 797-805.

Vaportzis E, Niechcial MA, Gow AJ (2019) A systematic literature review and meta-analysis of real-world interventions for cognitive ageing in healthy older adults. Ageing Research Reviews, 50: 110-30. 
Whitty E, Mansour H, Aguirre E, et al (2020) Efficacy of lifestyle and psychosocial interventions in reducing cognitive decline in older people: systematic review. Ageing Research Reviews, 62: 101113.

Winblad B, Amouyel P, Andrieu S, et al (2016) Defeating Alzheimer's disease and other dementias: a priority for European science and society. Lancet Neurology, 15: 455-32.
Wu L, Sun D (2017) Adherence to Mediterranean diet and risk of developing cognitive disorders: an updated systematic review and meta-analysis of prospective cohort studies. Sci Rep, 7: 41317.

Yu J-T, Xu W, Tan C-C, et al (2020) Evidence-based prevention of Alzheimer's disease: systematic review and meta-analysis of 243 observational prospective studies and 153 randomised controlled trials. Journal of Neurology, Neurosurgery \& Psychiatry, 91: 1201-9.

\section{MCQs}

Select the single best option for each question stem

1 In terms of cognitive change, abilities described as more age sensitive may be:

a verbal skills

b fluid abilities

c attention

d crystallised abilities

e all of the above.

\section{Different cognitive abilities develop and} change at different times and rates, but it has been suggested that there is shared variance in change across domains. In terms of cognitive change, this shared variance might account for:
a $0 \%$
b $20 \%$
c $40 \%$
d $60 \%$
e $80 \%$.

3 Cognitive ability and change have a heritable component. The genetic contributions might be described as:

a specific

b general

c highly polygenic

d marginal gains

e irrelevant.

4 In considering any factor as a potential determinant of cognitive ability or change, reverse causation:

a considers the stability of cognitive ability across the lifespan

b might reduce or remove certain reported associations between lifestyle and cognitive ability

c is difficult to consider as studies often do not have measures across time

d acknowledges that early ability predicts a range of lifestyle and behaviours across the lifespan

e all of the above.
5 The single most important factor for cognitive health is:
a sleep
b diet
c brain training
d physical activity
e none of the above. 